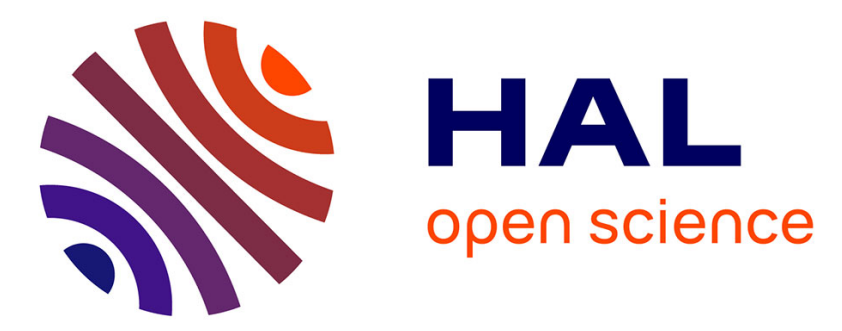

\title{
In situ study of spin waves in thin films deposited onto compliant substrates submitted to external stresses
} W Karboul-Trojet, D Faurie, Y Roussigné, S M Chérif, P.-O Renault, P. Goudeau

\section{- To cite this version:}

W Karboul-Trojet, D Faurie, Y Roussigné, S M Chérif, P.-O Renault, et al.. In situ study of spin waves in thin films deposited onto compliant substrates submitted to external stresses. Journal of Physics D: Applied Physics, 2011, 44 (15), pp.155002. 10.1088/0022-3727/44/15/155002 . hal-00609758

\section{HAL Id: hal-00609758 https://hal.science/hal-00609758}

Submitted on 20 Jul 2011

HAL is a multi-disciplinary open access archive for the deposit and dissemination of scientific research documents, whether they are published or not. The documents may come from teaching and research institutions in France or abroad, or from public or private research centers.
L'archive ouverte pluridisciplinaire HAL, est destinée au dépôt et à la diffusion de documents scientifiques de niveau recherche, publiés ou non, émanant des établissements d'enseignement et de recherche français ou étrangers, des laboratoires publics ou privés. 


\title{
In situ study of spin waves in thin film deposited onto
}

\section{compliant substrate submitted to external stresses}

\author{
W. Karboul-Trojet ${ }^{1}$, D. Faurie ${ }^{1}$, Y. Roussigné ${ }^{1}$, S. M. Chérif ${ }^{1, a}$, P.-O. Renault ${ }^{2}$, Ph. Goudeau ${ }^{2}$ \\ ${ }^{1}$ LSPM, UPR3407 CNRS, Université Paris13, 93430 Villetaneuse (FRANCE) \\ ${ }^{2}$ Institut Pprime, UPR 3346 CNRS-Université de Poitiers-ENSMA, 86962 Futuroscope
}

(FRANCE)

\footnotetext{
${ }^{a}$ Corresponding author: cherif@univ-paris13.fr
}

\begin{abstract}
We have developed a new methodology to study the effect of mechanical stress on spin waves in thin films deposited onto compliant substrates. It is based on micro-tensile tests combined with Brillouin light scattering spectroscopy, which allows in situ probing of the magnetization dynamics of the studied film upon deformation. This paper shows from both theoretical and experimental approaches that the magneto-elastic coupling in the saturation regime leads to a simple linear relationship between the spin waves frequency and the stress applied to the magnetic film. The linear part of the experimental data can be reproduced theoretically, assuming a complete strain transfer through the metallic film-compliant substrate interface.
\end{abstract}

PACS : 75.30.Ds , 75.40. Gb, 75.80.+q, 75.70. Ak, 75.50.cc 


\section{Introduction}

During the last decades, technological progress has been driven predominantly by the modern information and communication technology. The steadily increasing data output and functionality of devices have required an ongoing miniaturization of their structural elements. In the design and manufacturing of actual microelectronic and microelectromechanical systems (MEMS), thin metallic films play an important role. Noticeably, flexible and stretchable electronics are being developed for diverse applications, including electronic textiles [1], paper-like displays [2], and sensitive skins [3].

Thin films can show continuous geometry for which lateral dimensions are much higher than thickness (typically less than one micron), or show complex geometry with in plane discontinuities (wires or dots arrays) for which one or two lateral dimensions and thickness are of same order. Usually, thin films are deposited on substrates, which are several ten times thicker. They are more often submitted to temperature changes during fabrication and/or practical applications, which generate high mechanical stresses and affect the physical properties.

Few techniques have been developed to study the effects of applied stresses on magnetic properties of thin films. One approach has been proposed by McCord et al. in 2004, based on in situ analysis of static magnetization configuration by magneto-optical Kerr

microscopy (MOKE) [4]. Stress to the film-rigid substrate structure was applied with a bending fixture integrated in the sample holder of the microscope. Magnetoelastic effects were found to determine the domain distribution in the films. This effect has been used to test a stress sensor using magnetostrictive thin films [5]. On the other hand, such films deposited onto compliant substrates can be tensily stressed and in situ characterized by high-frequency (HF) permeability [6], MOKE [7] and giant magnetoresistance (GMR) measurements [8]. The main purpose of these studies was also to demonstrate the feasibility of a magnetostrictive 
sensor on a flexible polyimide substrate. The main advantage of the polyimide material is its high flexibility, which cannot be achieved by crystalline material, and its relatively low cost. Very recently, C. Barraud et al. showed that for magnetic tunnelling junctions (MTJs) grown on flexible organic substrates, the spin dependent tunnelling properties of spintronic devices can be preserved even after a relatively important (1\%) mechanical strain [9].

Dealing with the magnetization dynamics, one can report the work done by Weiler et al. on ferromagnetic resonance (FMR) of supported $\mathrm{Ni}$ thin films where the deformation was controlled by varying the applied voltage to the piezoelectric substrate [10]. The authors showed how magnetoelasticity allows switching the magnetic macroscopic easy axis in the $\mathrm{Ni}$ films by $90^{\circ}$ simply by changing the sign of the deformation. The in situ study of spin waves in thin films deposited on compliant substrate during mechanical tests is however still not usual.

The way proposed in this paper is to develop mechanical tests of ferromagnetic thin films on compliant substrates (with a micro-tensile tester), coupled in situ with Brillouin light scattering (BLS) technique, which allows measuring magnetization dynamics. We propose in this work to measure the frequencies of the spin waves as a function of the mechanical stress applied to polycrystalline Ni thin films. The analysis of their frequency variation under the applied external uniaxial stress should provide us with the knowledge of stress induced magnetic anisotropy change through an inverse magnetostrictive effect. A theoretical approach is proposed which allows deriving a simple relationship between the spin waves frequency and the stress applied to the magnetic film.

\section{Magnetic properties of as-deposited Ni film}

The Ni thin film was deposited by physical vapour deposition (PVD) technique on a dogbone polyimide (Kapton®) substrate. Polyimides are organic materials with an attractive 
combination of physical characteristics including low electrical conductivity, high tensile strength, chemical inertness, and stability at temperatures as high as $400{ }^{\circ} \mathrm{C}$. In addition to its widespread use in the microelectronics industry, Kapton ${ }^{\circledR}$ has an excellent thermal and radiation stability as evidenced by its routine use for vacuum windows at storage-ring sources. The base pressure of the deposition chamber was $7 \times 10^{-5} \mathrm{~Pa}$ while the working pressure during films growth was approximately $10^{-2} \mathrm{~Pa}$. The film deposition was made at room temperature with an $\mathrm{Ar}^{+}$-ion-gun sputtering beam at $1.2 \mathrm{keV}$. A slight $\{111\}$ texture has been evidenced by x-ray diffraction (not shown here) as often encountered in Ni films fabricated by PVD techniques. We used a Lake Shore 7404 vibrating sample magnetometer (VSM) in order to measure the magnetic hysteresis loop of the "as-deposited" sample. The apparatus shows a high sensitivity (100 nemu) and then enables to record extremely low magnetic signal. Figure 1 shows the obtained cycles for both in-plane (figure. 1(a)) and out-of-plane (figure. 1(b)) applied field. In figure. 1(a), we can see that the applied field for which the magnetization is uniform is about 300 Oe. Moreover, a weak coercive field is measured $(\approx 70$ Oe) related to the residual in-plane anisotropy of the film induced by the elaboration process. In figure. 1(b), the out-of-plane cycle is characteristic of hard axis loop. We measure a saturation field $\mathrm{H}_{\mathrm{s}}{ }^{\text {perp }}$ of about 4450 Oe corresponding to an effective magnetization $4 \pi \mathrm{M}_{\text {eff. }}$ Both cycles provide a saturation magnetization $4 \pi \mathrm{M}_{\mathrm{s}}$ equal to $6000 \mathrm{Oe}$.

We also determined the effective magnetization of the "as-deposited" Ni film, using micro-strip line ferromagnetic resonance (MS-FMR) technique. Figure 2 shows the frequency variation of the measured uniform mode with applied magnetic field $\mathrm{H}$. The resonance frequency is given, as a function of $\mathrm{H}$, by the usual expression [11]:

$$
\mathrm{f}=\frac{\gamma}{2 \pi}\left(\left(\mathrm{H}+\mathrm{H}_{/ /}\right)\left(\mathrm{H}+\mathrm{H}_{/ /}+4 \pi \mathrm{M}_{\text {eff }}\right)\right)^{1 / 2}
$$


Where $\gamma=1.846 .10^{2} \mathrm{GHz} / \mathrm{Oe}$ is the gyromagnetic factor. The in-plane anisotropy field $\mathrm{H}_{/ /}=$ 50 Oe and $4 \pi \mathrm{M}_{\text {eff }}=4300$ Oe are obtained from the best fit of the experimental data (see figure. 2). Both values well compare with the ones measured from VSM data. In the following, we use the value deduced from dynamic measurements to analyze the BLS results describing the stress effect on the spin waves frequencies. $4 \pi \mathrm{M}_{\text {eff }}$ differs from the bulk saturation magnetization $\left(4 \pi \mathrm{M}_{\mathrm{s}}=6000 \mathrm{Oe}\right)$ due to, as often reported for thin films, the existence of an initial uniaxial perpendicular anisotropy field $\mathrm{H}_{\perp}$. This perpendicular anisotropy originates from diverse contributions: initial internal stress, magnetocrystalline anisotropy, and a surface anisotropy that can be ignored in our case due to the thickness (200 $\mathrm{nm}$ ) of our sample. In the case of polycrystalline thin films deposited by PVD techniques, all these contributions generally yield a perpendicular contribution due to in-plane equibiaxial residual stress state, in-plane isotropic distribution of crystalline orientations. Thus this perpendicular anisotropy modifies the demagnetizing contribution yielding an effective demagnetizing field $4 \pi \mathrm{M}_{\text {eff }}=4 \pi \mathrm{M}_{\mathrm{s}}-\mathrm{H}_{\perp}$.

\section{Brillouin light scattering and in situ tensile testing}

Brillouin light scattering (BLS) spectroscopy is known as a powerful measurement tool, which allows for the local probing of magnetic dynamics [12-14]. Among the main advantages of this technique is the possibility to determine the frequency and the wave vector of magnetic excitations and to analyze the temporal evolution of magnetic processes with a typical time resolution below $1 \mathrm{~ns}$ [15-16]. The BLS technique is based on the spectral analysis of the laser light scattered by a magnetic sample. Due to the photon-magnon interaction, the scattered light also contains photons at frequencies shifted by the frequencies of magnons, in addition to the photons at the laser frequency. Consequently, analyzing these additional spectral components, a conclusion concerning frequencies and intensities of spin 
waves existing at probing light focused point of the sample surface (the probe size is about 100 microns) can be drawn. The measurements were achieved using a $(2 \times 3)$-pass tandem Fabry-Perot interferometer exhibiting a contrast and a finesse close to $10^{10}$ and 100 , respectively. The sample was illuminated by a single-mode $\mathrm{Ar}^{+}$ion laser at the wavelength $\lambda_{\text {laser }}=514.5 \mathrm{~nm}$. The spectra were achieved using crossed polarizations between the incident and the scattered beams, in order to take advantage of the selection rules for the magnon spectra and to prevent phonon lines to appear. A magnetic field $\mathrm{H}$ of $430 \mathrm{Oe}$, high enough to saturate the sample according to the in-plane saturation field determined from VSM loop, was applied parallel to the film plane and perpendicular to the incident beam. Reliable Brillouin spectra were obtained with recording times of 2 or $3 \mathrm{~h}$. We used a backscattering geometrical arrangement: the wave vectors of light were such as $\mathbf{k}_{\mathrm{s}}=-\mathbf{k}_{\mathrm{i}}$, where the subscripts i and s stand for incident and scattered, respectively. The in-plane wave vector $\mathbf{k}$ of the observed propagative excitations is given by $\mathbf{k}= \pm 2 \mathbf{k}_{\mathrm{i}}$ (where the symbols + and - hold for Stokes and anti-Stokes lines, respectively). Its amplitude $\mathrm{k}=2 \mathrm{k}_{\mathrm{i}} \sin \theta$ (where $\theta$ is the angle of incidence) can be modified by rotating the sample around the direction of the applied field; its order of magnitude is about $10^{5} \mathrm{~cm}^{-1}$.

In practical terms, performing tensile tests combined with BLS is straightforward since no rigid contact between the probe and the sample is needed, no specific sample geometry and preparation are required and the probed volume size of the sample is not needed to quantify the magnetic properties. It should be noted that BLS experiments have been performed using very low illuminating powers (less than $40 \mathrm{~mW}$ ) due to the low thermal conductivity of Kapton ${ }^{\circledR}$, in order to avoid undesirable heating of the film.

The tensile uniaxial loads were applied to dog-bone specimen (Ni film adherent to Kapton ${ }^{\circledR}$ substrate) by means of a $300 \mathrm{~N}$ Deben $^{\mathrm{TM}}$ tensile module (see figure. 3(a)). This tensile tester is equipped with a $75 \mathrm{~N}$ load cell fixed to one jaw enabling the force 
measurement with a precision of $0.2 \mathrm{~N}$. In order to estimate the stress concentration in the film, we assume that the in-plane macroscopic strains are transmitted through the filmsubstrate interface. In fact, as long as the elastic domain is scanned (several hundreds MegaPascals for nano-crystalline thin films), Geandier et al. showed that a complete strain transfer is achieved through a metallic film-compliant substrate interface, even for a nonreactive metal [17]. This result has an important implication since the modelling of the coated substrate (without adhesion interlayer) is much simplified considering a laminate formed by the film and the substrate. Using the formalism proposed by Renault et al. [18] and assuming that Poisson's ratio of the film $v_{\mathrm{f}}$ and the substrate $v_{\mathrm{s}}$ are similar $\left(v_{\mathrm{f}} \approx v_{\mathrm{s}} \approx 0.3\right)$, the longitudinal stress in the film is given by:

$$
\sigma_{x x}=\frac{\sigma^{\text {tot }} \cdot E_{f}}{E_{f} f_{f}+E_{s} f_{s}}
$$

Where $\sigma^{\text {tot }}$ is the total uniaxial stress applied to the film-substrate composite; $E_{f}, E_{s}$ and $f_{f}$, $f_{s}$ are the Young's modulus and thickness ratio of the film and the substrate respectively. The thickness ratios are defined as follows:

$$
f_{f}=\frac{t_{f}}{t_{f}+t_{s}} \text { and } f_{s}=\frac{t_{s}}{t_{f}+t_{s}}
$$

with $t_{f}$ and $t_{s}$ the film and substrate thicknesses respectively. Assuming the following parameters $\left(E_{\mathrm{f}}=200 \mathrm{MPa}, \mathrm{E}_{\mathrm{s}}=3.8 \mathrm{MPa}, \mathrm{t}_{\mathrm{f}}=200 \mathrm{~nm}, \mathrm{t}_{\mathrm{s}}=125 \mu \mathrm{m}\right.$ and width of the sample $\mathrm{w}=6 \mathrm{~mm}$ ), a 1 Newton applied load to the film-substrate composite leads to a longitudinal stress $\sigma_{\mathrm{xx}}=64.8 \mathrm{MPa}$ in the thin film. Moreover the in-plane transversal stress can be neglected here since $v_{\mathrm{f}}=v_{\mathrm{s}}$. Notice that the maximum applied strain in this work is estimated to be $0.1 \%$ so that we can infer no damage of the sample.

The evolution of the so-called Damon-Eshbach (DE) dipolar surface mode frequency [19] (configuration where the spin wave propagation direction is perpendicular to the static 
magnetization at saturation, i.e. to $\mathbf{H}$, see figure. 3(b)) has been studied by BLS. The strength of the applied magnetic field in the direction parallel to the uniaxial stress is $\mathrm{H}=430$ Oe. It is sufficient to saturate the Ni film when no stress is applied. The measured decrease of the DE mode frequency with increasing stress is shown in figure. 4 and will be discussed afterwards. We noticed the large asymmetry of the Stokes/anti-Stokes spectrum which affects the DE line: we checked that the asymmetry is reversed when the direction of $\mathbf{H}$ is reversed, as it should be. However, due to the very low illuminating powers (less than $40 \mathrm{~mW}$ ), we did not clearly observe the standing spin waves (SSW) lines which, as usually reported, show weaker intensity regarding to the DE mode [20-21].

\section{Results and discussion}

When the effect of external mechanical stresses is scrutinized, the magnetic anisotropy constant $(\mathrm{K})$ that comes from the magnetoelastic coupling can be expressed as follows, with $\lambda$ the magnetostriction coefficient at saturation [22]:

$$
\mathrm{K}=\frac{3}{2} \lambda \sigma_{\mathrm{xx}}
$$

The magnetic energy reads:

$$
\begin{array}{r}
\mathrm{E}=\mathrm{E}_{\text {zeeman }}+\mathrm{E}_{\text {demagnetizing }}+\mathrm{E}_{\text {external stress }}+\mathrm{E}_{\text {anisotropy }} \\
\text { With }\left\{\begin{array}{l}
\mathrm{E}_{\text {zeeman }}=-\mathrm{HM}_{\mathrm{x}} \\
\mathrm{E}_{\text {demagnetizing }}=2 \pi \mathrm{M}_{\mathrm{y}}{ }^{2} \\
\mathrm{E}_{\text {external stress }}=-\frac{\mathrm{KM}_{\mathrm{x}}^{2}}{\mathrm{M}_{\mathrm{s}}^{2}} \\
\mathrm{E}_{\text {anisotropy }}=-\frac{\mathrm{K}_{0} \mathrm{M}_{\mathrm{y}}^{2}}{\mathrm{M}_{\mathrm{s}}^{2}}-\frac{\mathrm{K}_{0}^{\prime} \mathrm{M}_{\mathrm{x}}^{2}}{\mathrm{M}_{\mathrm{s}}^{2}}
\end{array}\right.
\end{array}
$$


Where $\mathrm{x}$ is the direction of the applied field as well as the direction of the applied stress and where $\mathrm{y}$ is the normal to the layer as shown in figure $3(\mathrm{~b}) . \mathrm{K}_{0}$ and $\mathrm{K}_{0}^{\prime}$ are the initial perpendicular and parallel anisotropy constants respectively. It should be noted here that the effective demagnetizing field reads $4 \pi \mathrm{M}_{\text {eff }}=4 \pi \mathrm{M}_{\mathrm{s}}-\frac{2 \mathrm{~K}_{0}}{\mathrm{M}_{\mathrm{s}}}$ as discussed above with $\mathrm{H}_{\perp}=$ $\frac{2 \mathrm{~K}_{0}}{\mathrm{M}_{\mathrm{s}}}$. Moreover the in-plane anisotropy field is expressed as: $\mathrm{H}_{/ /}=\frac{2 \mathrm{~K}_{0}^{\prime}}{\mathrm{M}_{\mathrm{s}}}$

The external stress contribution yields a field $H_{\sigma}=-\frac{\partial E_{\text {externalstress }}}{\partial M_{x}}=\frac{2 K M_{x}}{M_{s}^{2}}$. This field is added to the applied field $H=-\frac{\partial E_{\text {Zeeman }}}{\partial M_{x}}$. In the case of a saturated film $M_{x}=M_{s}$, the effective field becomes $\mathrm{H}_{\text {eff }}=\mathrm{H}+\mathrm{H}_{/ /}+\mathrm{H}_{\sigma}$, with $\mathrm{H}_{\sigma}=\frac{2 \mathrm{~K}}{\mathrm{M}_{\mathrm{s}}}$.

The frequency of the DE surface mode is given, in the dipolar approximation and neglecting anisotropy, by the well-known expression [23]:

$$
f=\frac{\gamma}{2 \pi}\left(\left(H+2 \pi M_{s}\right)^{2}-\left(2 \pi M_{s}\right)^{2} \exp \left(-2 k t_{f}\right)\right)^{1 / 2}
$$

where $t_{\mathrm{f}}$ is the thickness of the magnetic film and $\mathrm{k}$ the wave vector norm of the magnetic excitation. $\mathrm{k}$ is determined by $\mathrm{k}=\left(\frac{4 \pi}{\lambda_{\text {laser }}}\right) \sin \theta$, with $\theta=45^{\circ}$.In our case, the exponential term is about $10^{-3}$ so that it can be neglected. Thus, the DE mode frequency reads:

$$
\mathrm{f}=\frac{\gamma}{2 \pi}\left(\mathrm{H}+2 \pi \mathrm{M}_{\mathrm{s}}\right)
$$

In our case the effective demagnetizing field $4 \pi \mathrm{M}_{\text {eff }}$ replaces the demagnetizing field $4 \pi \mathrm{M}_{\mathrm{s}}$.

Moreover the effective field $\mathrm{H}_{\text {eff }}=\mathrm{H}+\frac{2 \mathrm{~K}_{0}^{\prime}}{\mathrm{M}_{\mathrm{s}}}+\frac{2 \mathrm{~K}}{\mathrm{M}_{\mathrm{s}}}$ replaces the applied field $\mathrm{H}$. Therefore, following equation (4), we obtain 


$$
\mathrm{f}=\frac{\gamma}{2 \pi}\left(\mathrm{H}+\frac{2 \mathrm{~K}_{0}^{\prime}}{\mathrm{M}_{\mathrm{s}}}+\frac{3 \lambda}{\mathrm{M}_{\mathrm{s}}} \sigma_{\mathrm{xx}}+2 \pi \mathrm{M}_{\mathrm{eff}}\right)
$$

If $\lambda$ is negative (case of $\mathrm{Ni}$ ), the magnitude of the effective field, decreases when increasing the applied stress $\sigma_{\mathrm{xx}}$. Figure 5 shows the evolution of the DE mode frequency as a function of the applied stress for the applied field $H=430$ Oe. One can remark that the frequency decreases linearly when increasing the stress $\sigma_{x x}$ in the saturation regime as predicted by equation (9). Such a variation is observed below a given value of the applied stress (position of the vertical dashed line on the figure 5). Above this value the evolution changes denoting a new frequency evolution regime. This transition value separates the saturated and the non saturated magnetization configurations and corresponds to the situation where the applied magnetic field is exactly compensated by the stress-induced anisotropy magnetic field, i.e. when the effective field value is zero. The straight line in figure 5 show the theoretical evolution in the saturation configuration using the effective magnetostriction coefficient of the Ni film at saturation, usually reported in the literature $\left(\lambda=-37 \times 10^{-6}\right)$ [22]. It should be noted that the $\sigma_{\mathrm{c}}=-\frac{\left(\mathrm{H}+\mathrm{H}_{/ /}\right) \mathrm{M}_{\mathrm{s}}}{3 \lambda} \approx 200 \mathrm{MPa}$ value of the critical stress estimated using this $\lambda$ value agrees with the measured one shown by the dashed vertical line of figure 5 . For higher values of the applied stress, the magnetization may change its direction in the film plane by a rotation towards the easier direction perpendicular to that of the applied stress and that of applied magnetic field, leading to a non saturated magnetization state. For this configuration, calculations are in progress for deriving an expression describing the frequency evolution. It's to notice that similar effects have been observed from the investigation of the dependency between strain and permeability in optimized magnetoelastic films deposited onto polyimide substrates for sensor applications in the lower GHz range [6]. Finally we checked that when the stress is removed, the DE mode frequency returns to its initial value. 
Let's mention that if the stress is applied perpendicular to the direction of the magnetic field, it can be shown that the DE mode frequency evolution versus the stress is also linear ; however the slope sign is opposite regarding to the parallel configuration and its magnitude is twice smaller.

\section{Conclusion}

The effect of mechanical stress on spin waves in thin films deposited onto compliant substrates has been studied using a new approach based on mechanical tests coupled in situ with Brillouin light scattering (BLS) technique. From the experimental data we deduced the effective magnetostriction coefficient at saturation of the film. We found theoretically that, when the directions of the applied stress and that of the applied magnetic field are parallel, the expression of the spin waves frequency in the Damon-Eshbach (DE) configuration is only modified by the addition of an anisotropy term induced by the magneto-elastic coupling.

\section{Acknowledgments}

This work has been developed in the frame of the French Research Agency (ANR) in the JCJC-SIMI9 program (project $n^{\circ}$ ANR 2010 JCJC 090601 entitled "SpinStress") and of the Cnano Ile-de-France (project IMADYN). The authors would like to thank Pr. Frédéric Mazaleyrat for the VSM measurements. 


\section{References}

[1] Gu J F, Gorgutsa S, Skorobogatiy M 2010, Smart Mater. Struct. 19, 115020

[2] Fortunato E, Correia N, Barquinha P, Pereira L, Goncalves G, Martins R 2008, IEEE Elec. Dev. Lett. 29, 988

[3] Xiao S Y, Che L F, Li X X, Wang Y L 2007, Microelec. J. 38, 360

[4] McCord J, Schafer R, Frommberger M, Glasmachers S, Quandt E 2004, J. Appl. Phys. 95, 6861

[5] Frommberger M, Glasmachers S, Schmutz C, McCord J, Quandt E 2005, IEEE Trans. Magnet. 41, 6391

[6] Ludwig A, Tewes M, Glasmachers M, Löhndorf M, Quandt E 2002, J. Magn. Magn. Mater. 242-245, 1126

[7] Ozkaya B, Saranu S R, Mohanan S, Herr U 2008, Phys. Stat. Sol. A 205 (8), 1876

[8] Uhrmann T, Bar L, Dimopoulos T, Wiese N, Ruhrig M, Lechner A 2006, J. Magn. Magn. Mater. 307, 209

[9] Barraud C, Deranlot C, Seneor P, Mattana A, Dlubak B, Fusil S, Bouzehouane K, Deneuve D, Petroff F,.Fert A 2010, Appl. Phys. Lett. 96, 072502

[10] Weiler M, Brandlmaier A, Geprägs S, Althammer M, Opel M, Bihler C, Huebl H,. Brandt M S, Gross R, Goennenwein S T B 2009, New J. Phys. 11, 013021

[11] Kittel C 1948, Phys. Rev. 73, 155

[12] Demokritov S O and Tsymbal E 1994, J. Phys. C6, 7145

[13] Chérif S M, Roussigné Y, Sergeeva N A, Moch P 2005, J. Appl. Phys. 98, 063905

[14]Zighem F, Roussigné Y, Chérif S M, Moch P 2007, J. Phys.: Condens. Matt. 19, 176220

[15] Bauer M, Büttner O, Demokritov S O, Hillebrands B, Grimalsky V, Rapoport Yu, Slavin A N 1998, Phys. Rev. Lett. 81, 3769 
[16] Serga A A, Hillebrands B, Demokritov S O, Slavin A N, Wierzbicki P, Vasyuchka V, Dzyapko O, Chumak A 2005, Phys. Rev. Lett. 94, 167202

[17] G. Geandier, Renault P O, Le Bourhis E, Villain P, Goudeau Ph, Faurie D, Le Bourlot C, Djemia Ph, Castelnau O, Chérif S M 2010, Appl. Phys. Lett. 96, 041905

[18] Renault P O, Le Bourhis E, Villain P, Goudeau Ph,. Badawi K F, Faurie D 2003, Appl. Phys. Lett. 83, 473

[19] Damon R W and Eshbach J R 1961, J. Phys. Chem. Solids 19, 308

[20] Camley R E and Mills D L 1978, Phys. Rev. B 18, 4821

[21] Cochran J F and Dutcher J R 1988, J. Magn. Magn. Mater. 73, 299

[22] Du Tremolet De Lacheisserie E 1993, Magnetostriction. Theory and applications of magnetoelasticity (Edit. CRC Press Inc).

[23] Eshbach J R and Damon R W 1960, Phys. Rev. 118, 1208 


\section{Figure captions}

Figure 1. VSM magnetic hysteresis loop of the "as-deposited" sample with (a) applied field in the tensile direction (in-plane) and (b) applied field in the out-of-plane direction. This measurement provides an effective magnetization $4 \pi \mathrm{M}_{\text {eff }}=4450$ Oe.

Figure 2. Resonance frequency as a function of the applied magnetic field H. The best fit (continuous line) of the experimental data (symbol) provides an effective magnetization $4 \pi \mathrm{M}_{\text {eff }}=4300$ Oe in relative accordance with VSM measurements.

Figure 3. (a) Image of the experimental set-up. The micro-tensile tester is placed into the electro-magnet, while the laser beam is focused on the sample. Here, the direction of magnetic field is along the tensile axis. (b) Sketch of the experimental set-up. The tensile stress $\sigma^{\text {tot }}$ applied to the film-substrate composite, as well as the applied field $\mathbf{H}$, is along the $\mathbf{x}$-axis direction, leading to a longitudinal stress $\sigma_{x x}$ in the thin film. In the used Damon-Eshbach (DE) configuration, the wave vector $\mathbf{k}$ is along the $\mathbf{z}$-axis direction (perpendicular to $\mathbf{H}$ and lying in the film plane).

Figure 4. Evolution of the BLS frequency shift for different applied longitudinal stresses $\sigma_{\mathrm{xx}}$. The measured magnon frequency decreases when increasing applied stress.

Figure 5. DE spin wave frequency as a function of $\sigma_{x x}$. The continuous straight line shows the adjustment based on equation. (9) to the experimental data for the magnetization saturation configuration, delimited by the dashed vertical line, where the anisotropy field 
induced by the external stress $H_{\sigma}$ is lower than the sum of applied magnetic field $\mathrm{H}$ and initial in-plane anisotropy field $\mathrm{H}_{/ /}$. 

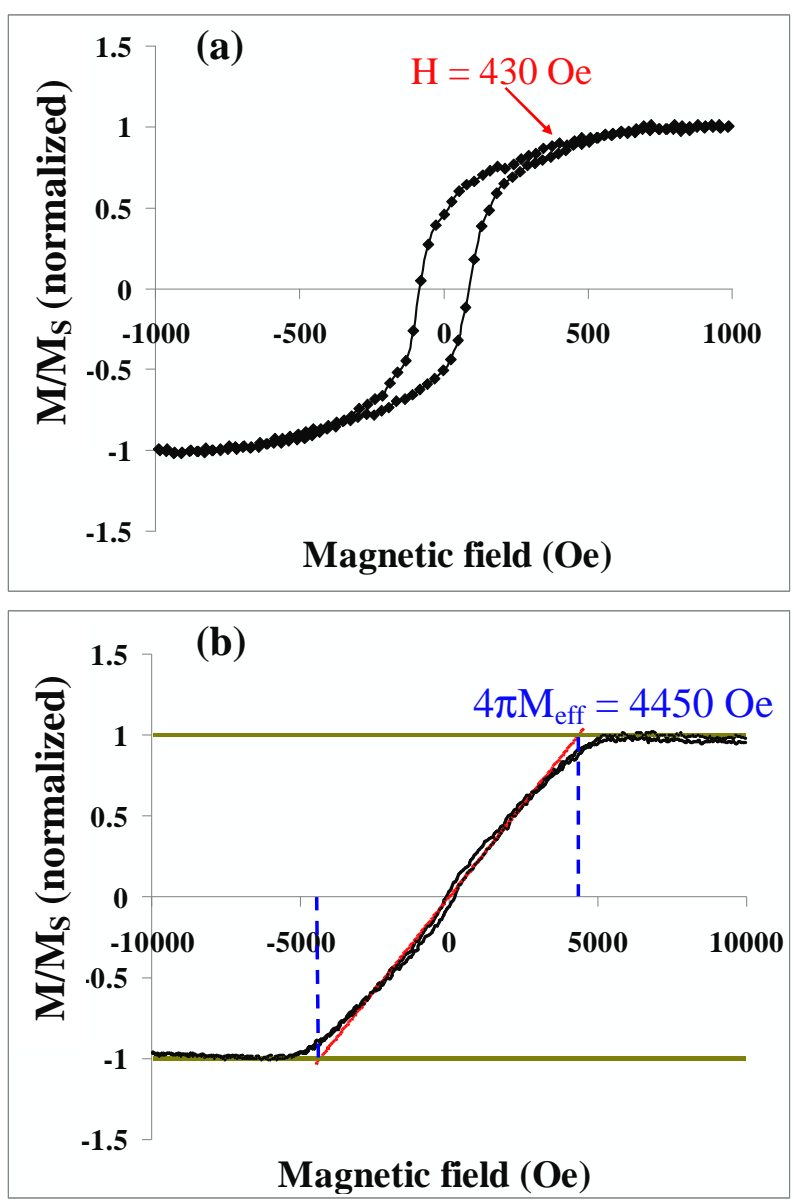

FIG. 1 Karboul et al. 


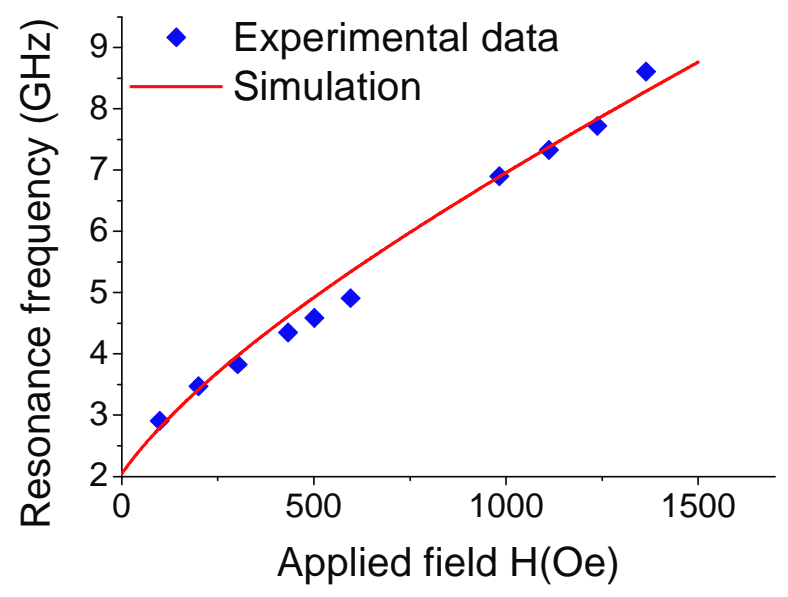

FIG. 2 Karboul et al. 

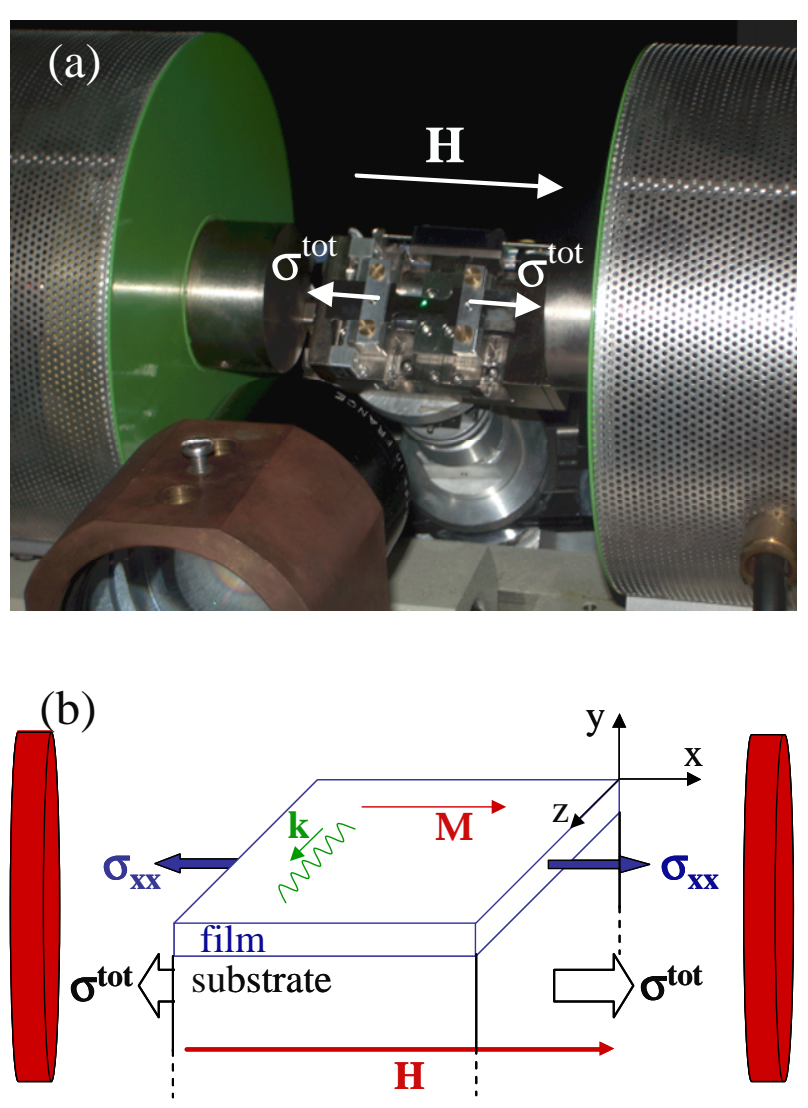

FIG. 3 Karboul et al. 


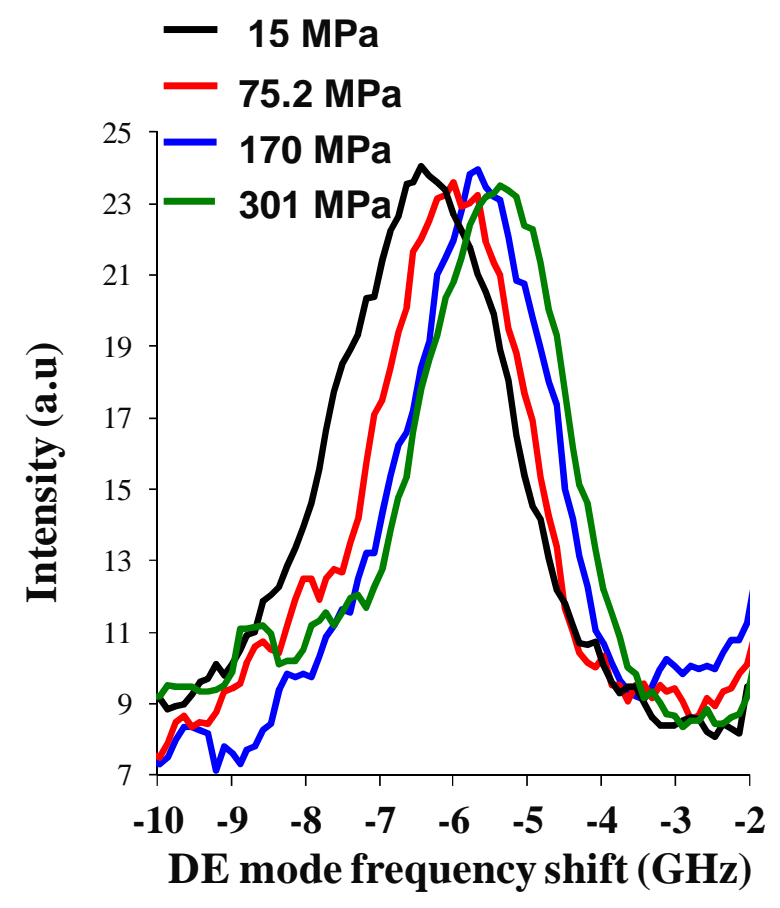

FIG. 4 Karboul et al. 


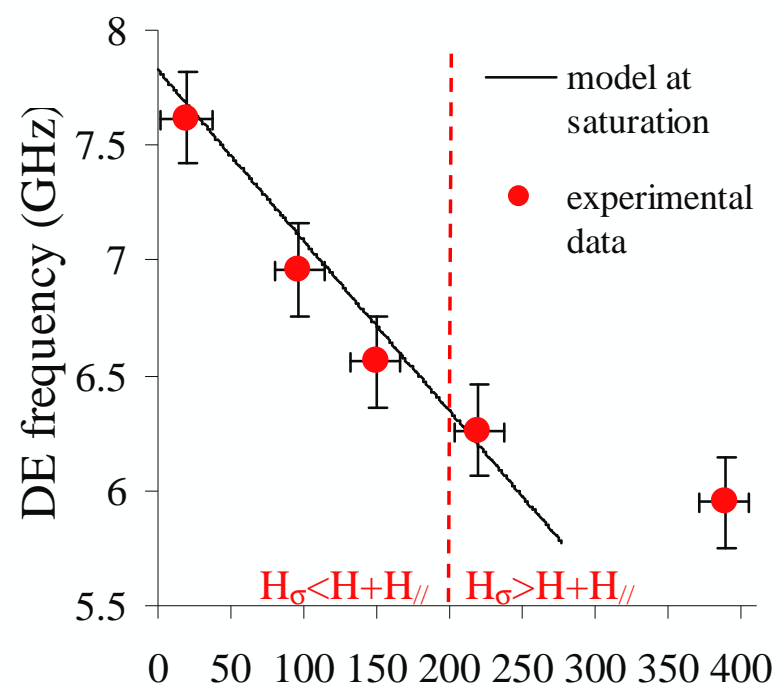

Applied stress $\sigma(\mathrm{MPa})$

FIG. 5 Karboul et al. 